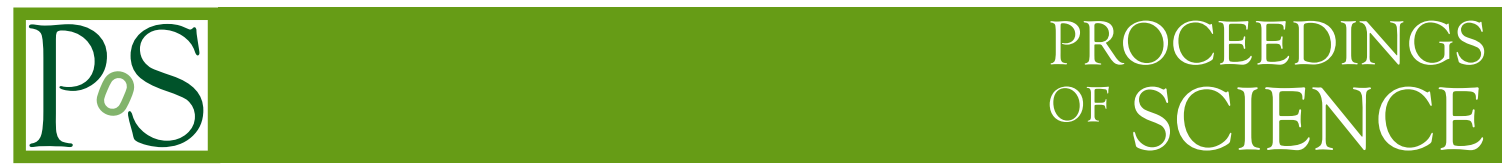

\title{
Searches for long-lived particles with the ATLAS detector
}

\author{
Andrew Haas* \\ On behalf of the ATLAS Collaboration \\ New York University \\ E-mail: Andy . Haas enyu . edu
}

Several extensions of the Standard Model predict the existence of new, massive, long-lived particles. We report on searches for particles leaving disappearing tracks in the outer tracker, weaklyinteracting long-lived particles decaying to collimated lepton-jets far away from the interaction point, slow and highly ionizing particles traversing the detector, and highly-ionizing magnetic monopoles. Results of analyses using data recorded in 2011 at $\sqrt{s}=7 \mathrm{TeV}$ center-of-mass energy by the ATLAS experiment at the LHC are shown.

36th International Conference on High Energy Physics,

July 4-11, 2012

Melbourne, Australia

\footnotetext{
* Speaker.
} 


\section{Introduction}

New, massive, long-lived particles are predicted by countless extensions of the Standard Model (SM) [1]. The Large Hadron Collider (LHC) collided protons at $\sqrt{s}=7 \mathrm{TeV}$ in 2011 with high luminosity, possibly allowing the production of such particles. The ATLAS experiment [2] is a multi-purpose particle detector at the LHC and recorded approximately $5 \mathrm{fb}^{-1}$ of proton-proton collisions in 2011.

Depending on the properties of the new, long-lived particle, its typical lifetime, and how it dominantly decays to SM particles, various signatures in the ATLAS detector are possible. Decays can be observed in the inner tracking system, outer tracking system, calorimeters, or muon system. The signatures of long-lived particles in these systems include:

- displaced vertices where reconstructed charged-particle tracks overlap

- disappearing tracks (when the charged long-lived particle decays to a neutral particle and a very low transverse momentum charged particle)

- charged tracks that are measured to travel significantly slower than the speed of light

- charged tracks that deposit significantly more charge per unit distance than a standard particle (highly ionizing due to its high mass, large charge, and/or slow speed)

- long-lived particles that stop in the dense detectors (calorimeters) and decay much later during accelerator bunch-crossings with no colliding protons

Given the unusual signatures involved, searches for these new, long-lived particles often involve special trigger algorithms to record the events efficiently, special data-processing techniques to maintain the required detailed event information that is typically discarded, special reconstruction algorithms, special programs for the simulation of the new, long-lived particle production, interactions in the detector, and decays, and methods for estimating novel cosmic and detector backgrounds.

\section{Disappearing track search}

Supersymmetry offers many very well-motivated long-lived particles. A particularly attractive scenario is the chargino becoming long-lived because it is nearly degenerate in mass with the neutralino (dark matter), since only the self-energy of the electro-magnetic interaction separates the two states. This is often realized in anomaly-mediated supersymmetry-breaking models. The chargino can be produced either directly through electroweak interactions, or in the decays of heavier, strongly-produced supersymmetric particles. The chargino can travel $\approx 10 \mathrm{~cm}$ for a lifetime of $\approx 1 \mathrm{~ns}$, before decaying into a neutralino which escapes the detector unseen and a low-momentum $(<1 \mathrm{GeV})$ charged pion (or electron or muon, more rarely). The low momentum charged track is difficult to reconstruct, due to its very large distance from the primary interaction point and small number of interactions in the tracking system. But the original, high-momentum, charged track from the chargino is not seen in the tracking layers past its decay point - i.e. a disappearing track (see Fig. 1, left). Backgrounds to such a signature exist from charged particles suffering hadronic 
interactions in the tracker material and from poorly reconstructed low-momentum tracks due to large-angle scatterings.
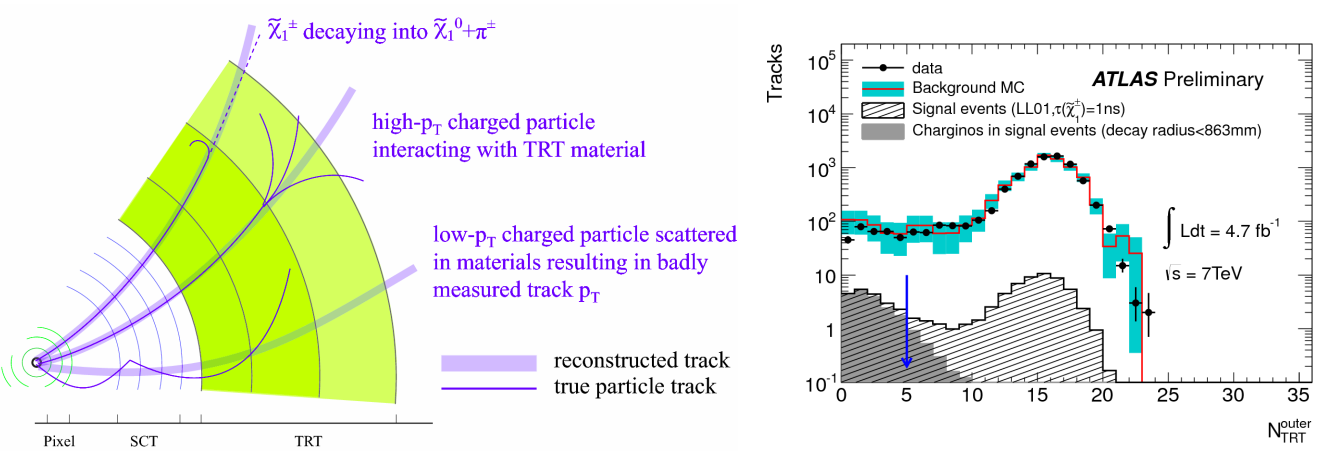

Figure 1: (From the disappearing track search [3].) Left: Diagram of signal and background track processes in the ATLAS tracker for the disappearing track search. Right: The number of TRT (outer-tracker) hits expected for background and signal tracks, compared to data.

ATLAS has performed a search for such disappearing tracks using $4.7 \mathrm{fb}^{-1}$ of $2011 \mathrm{p}$-p data at $\sqrt{s}=7 \mathrm{TeV}$ [3]. Events are triggered using a jet with large transverse momentum, $p_{T}>75 \mathrm{GeV}$ and missing transverse momentum, $M E_{T}>55 \mathrm{GeV}$. Offline, at least 3 jets are required with $p_{T}>$ $130,60,60 \mathrm{GeV}$ and $M E_{T}>130 \mathrm{GeV}$. The analysis presented here thus focuses on the stronglyproduced squarks/gluinos which then decay to the long-lived charginos through cascades involving jets. Leptons are vetoed to reduced the $\mathrm{W}+$ jets background, which leads to high-momentum leptons and large $M E_{T}$. The high- $p_{T}$ chargino track is required to have $p_{T}>10 \mathrm{GeV}$ as a preselection and be isolated from other tracks in a cone of $\Delta R>0.1$. Finally, less than 5 hits are required to be present on the track in the outer layer of the tracker (see Fig. 1, right).
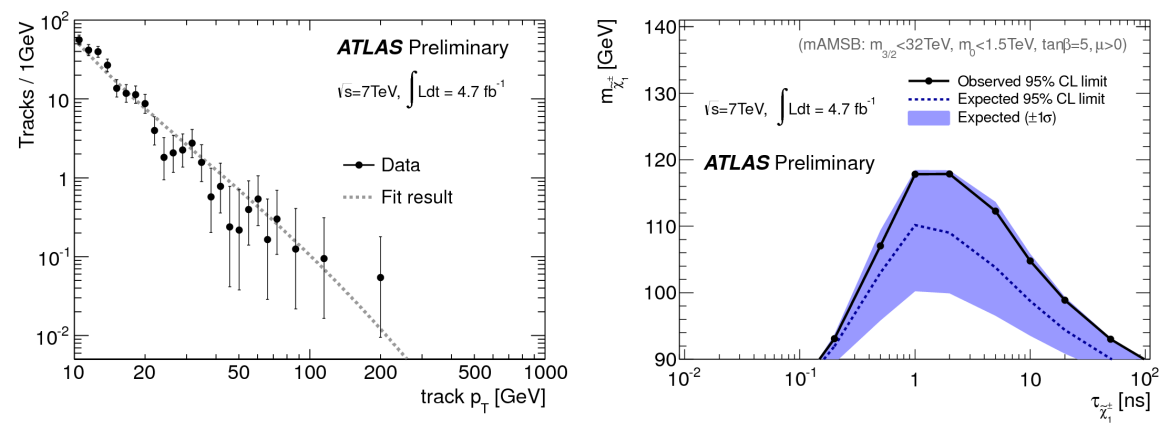

Figure 2: (From the disappearing track search [3].) Left: the track $p_{T}$ for data candidates compared to a fit to a sum of background and signal track $p_{T}$ spectra. Right: Limits on the chargino mass vs. chargino lifetime.

Background templates are derived for the hadronic-interaction track and badly-reconstructed track backgrounds, which are then fit to the data track $p_{T}$ spectrum from the 304 candidate events, along with a signal template (which peaks at high- $p_{T}$ ) (see Fig. 2, left). No contribution from the signal template is preferred, so limits are set on the signal production, as a function of the chargino 
lifetime and mass (see Fig. 2, right). The limit on the chargino mass is extended up to $118 \mathrm{GeV}$ for a lifetime of $1 \mathrm{~ns}$, above the LEP limit of $92 \mathrm{GeV}$.

\section{Search for lepton-jets in the muon system}
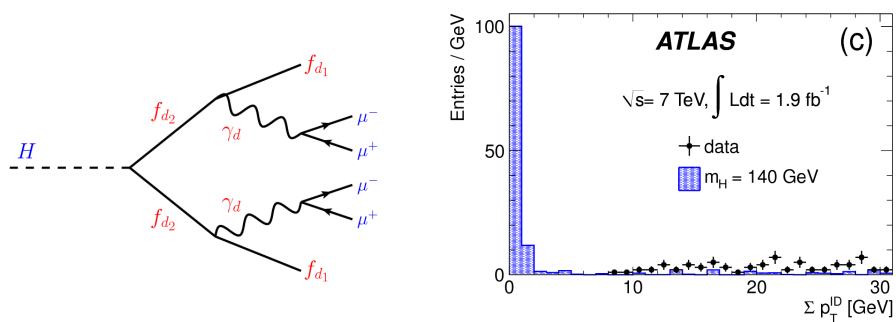

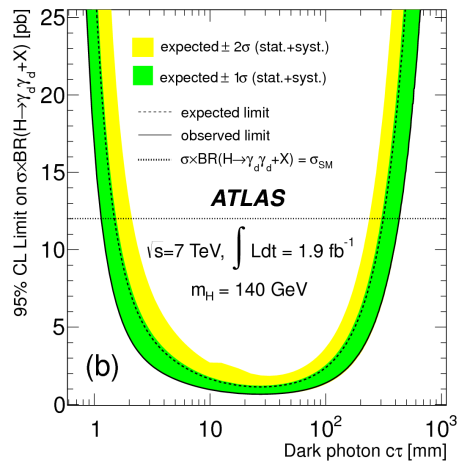

Figure 3: (From the lepton-jets search [4].) Left: Feynman diagram for the signal process of two lepton-jets from long-lived dark-photons. Center: Track isolation for data events and simulated signal events passing all other selections. Right: Limits on the dark-photon production as a function of the dark-photon lifetime.

Another analysis searched for lepton-jets (groups of collimated leptons, and possibly some pions) occurring in the muon system, from the long-lived decays of new, neutral, long-lived particles (called dark-photons) produced in e.g. Higgs decays [4] (see Fig. 3, left). Events are triggered requiring at least 3 muons in the muon system with $p_{T}>6 \mathrm{GeV}$, which means that one lepton-jet in the event must give two trigger muons, even at the first trigger level. The efficiency for this trigger is approximately $30 \%$, averaged over $p_{T}$ and $\eta$ of the lepton jets. Offline, muon lepton-jets are reconstructed using a cone algorithm of size $\mathrm{R}=0.2$, with at least 2 muons per lepton-jet. Each event requires at least two such lepton-jets, which must also be isolated in the calorimeter and tracker to reduce QCD jet backgrounds (see Fig. 3, middle).

No events are observed passing all selections in $1.94 \mathrm{fb}^{-1}$ of $2011 \mathrm{p}-\mathrm{p}$ data, so limits are set on the production as a function of the dark-photon particle lifetime, for various Higgs boson masses (see an example in Fig. 3, right). For a $10 \%$ branching ratio of Higgs to dark-photons, proper lifetimes between 5 and $100 \mathrm{~mm}$ can be excluded at 95\% C.L. (the dark-photon has a small mass, $\approx 1 \mathrm{GeV}$, thus has a large boost when produced from Higgs decay).

\section{Search for massive, slow, highly-ionizing tracks}

A very general search has been performed for long-lived, massive, charged tracks, from new particles such as long-lived squarks, gluinos, or staus [5]. Massive particles are expected to be produced with speed significantly smaller than the speed of light. The speed of the particle can be directly measured using timing information from several detectors, such as calorimeters and muon systems. The slow speed also causes the particle to deposit more charge per unit length in the detectors, due to the Bethe-Block relations, which can be measured particularly well in the pixel tracking detector. 

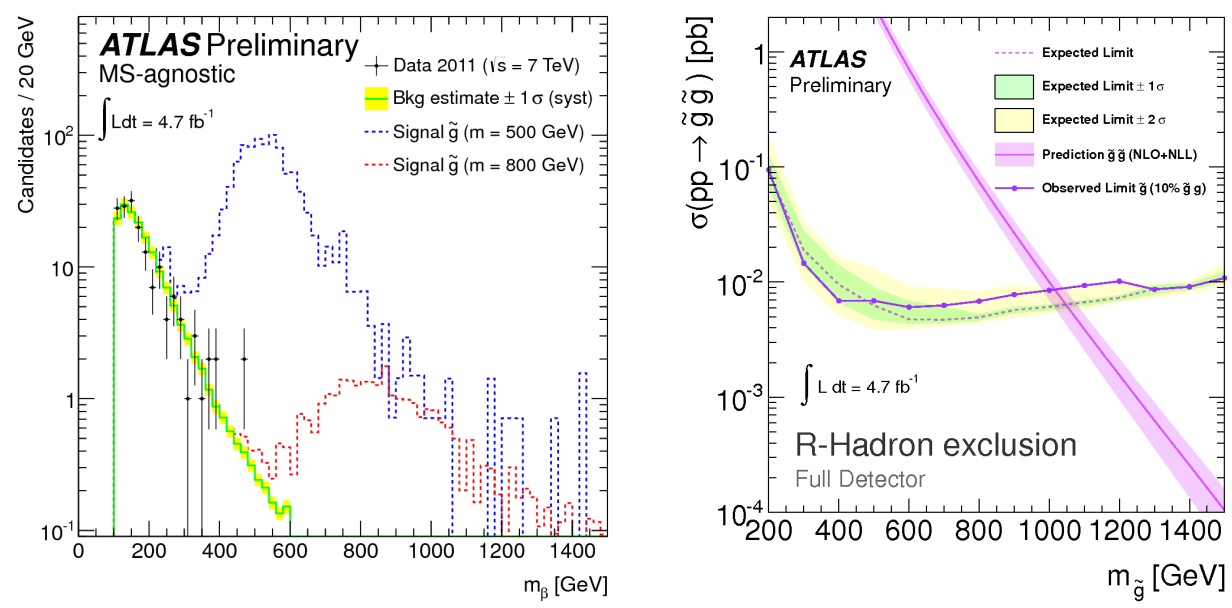

Figure 4: (From the massive, slow, long-lived particle search [5].) Left: The mass of each candidate track in data and from backgrounds in one of the massive, slow, long-lived particle searches, reconstructed using information from the tracker and calorimeters only. Right: An example of limits set in the massive, slow, long-lived particle searches, as a function of the long-lived particle mass.

Three searches are actually performed, each optimized towards different possible signals. The first search is optimized for colorless particles such as stau, which is guaranteed to be charged through the whole detector and escape. It uses an inner-detector charged track, plus calorimeter and muon measurements of timing. Since the selection is very efficient, two tracks in the same events are also looked for, in addition to the single track search. The second search is optimized for colored particles such as squarks or gluinos (R-hadrons), which may become neutral in the calorimeters from interactions with detector material. Only an inner-detector charged track and calorimeter information is used for this search. Lastly, shorter lifetime particles may decay before reaching the calorimeter or outer-tracker. So this last search uses only a silicon track and the ionization measurement in the pixel silicon detector. Either a single-muon or $M E_{T}$ trigger is used, depending on whether the track is assumed to reach the muon system in time or not, and is typically 90\% efficient for stau and 15-40\% efficient for R-hadrons.

The data are compared to models of background derived from data, given measured amounts of timing and ionization mis-measurements based on studies of $\mathrm{Z}$ decays. No excess is observed at high mass (large ionization and/or slow time) for any of the searches, so limits are placed on the particles' masses (see Fig. 4). The long-lived stau is excluded at 95\% C.L. below $310 \mathrm{GeV}$, gluinos below $985 \mathrm{GeV}$, and stop/sbottom below about $600 \mathrm{GeV}$.

\section{Search for very highly-charged particles (monopoles)}

Lastly, ATLAS has also searched for production of very highly-charged, long-lived, particles such as magnetic monopoles [6]. The signature is large ionization in the tracker from the large charge (68.5e for a Dirac monopole) and energy deposited in the electromagnetic calorimeter. The events are triggered requiring an electromagnetic calorimeter cluster with $E_{T}>60 \mathrm{GeV}$. Offline, a road is defined around the track from the cluster to the primary p-p interaction along which high- 
threshold hits are searched for from $\delta$-rays ejected by the highly-charged track (see Fig. 5, left). At least 20 such hits are required, and at least $70 \%$ of the hits on the track must also be highthreshold. The electromagnetic shower in the calorimeter is also expected to be very narrow, due to the suppression of Bremsstrahlung and pair-production due to the large monopole mass. Fig. 5, center, shows the shower width $\left(\sigma_{R}\right)$ expected in signal (red) compared to showers seen in data. A cut requires signal candidates to have $\sigma_{R}<0.017$.
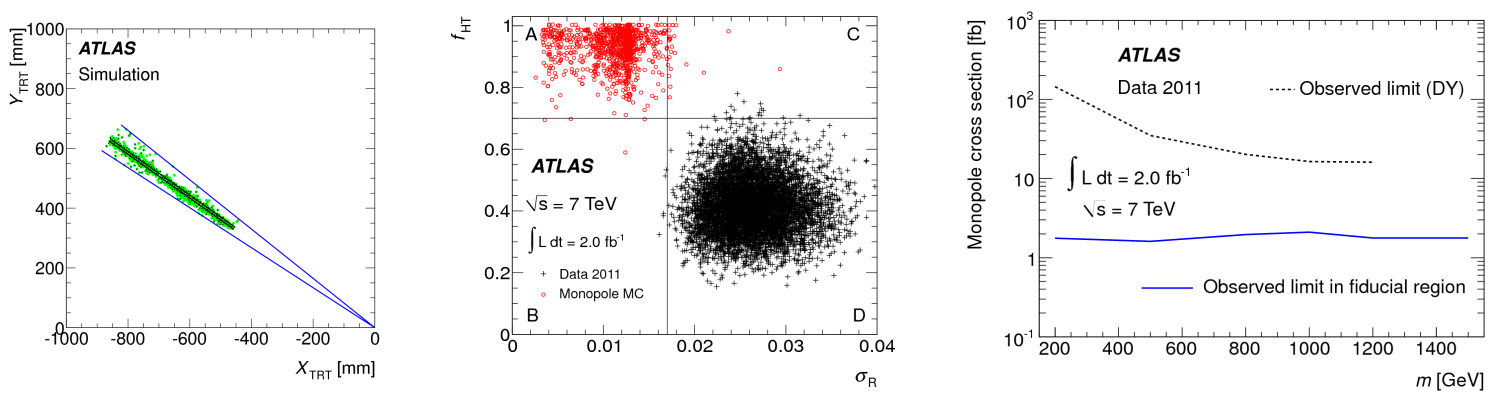

Figure 5: (From the monopole search [6].) Left: The road around the monopole in which high-threshold hits are searched for in the highly-ionizing track search. Middle: The fraction of high-threshold hits vs. the electromagnetic calorimeter cluster width for monopoles (red) vs. data candidates (black). Right: Limits on the monopole cross-section vs. monopole mass.

No candidates are observed in $2.0 \mathrm{fb}^{-1}$ of $2011 \mathrm{p}$-p data, with $0.011 \pm 0.007$ events expected from background. Limits are thus set on the monopole production cross-section as a function of the monopole mass (see Fig. 5, right). A smaller fraction of events are produced in the fiducial region at low mass and escape into the forward regions, but the analysis is just as sensitive to the low mass monopoles that do fall within the fiducial region. Monopole cross-sections above $\approx 20 \mathrm{fb}$ are excluded at $95 \%$ C.L. for a monopole mass of $1 \mathrm{TeV}$, the first such constraints from the LHC.

\section{Summary}

Several searches for new, long-lived particles using 2011 data from the ATLAS detector have been described. No evidence for such particles has been observed using this data set. Searches for this phenomena and other additional signatures of long-lived particles will continue using a much larger data set (and at $\sqrt{s}=8 \mathrm{TeV}$ ) in 2012 .

\section{References}

[1] M. Fairbairn, A. C. Kraan, D. A. Milstead, T. Sjostrand, P. Z. Skands and T. Sloan, Phys. Rept. 438, 1 (2007) [hep-ph/0611040].

[2] ATLAS Collaboration, 2008 JINST 3 S08003.

[3] ATLAS Collaboration, ATLAS-CONF-2012-034, https://cdsweb.cern.ch/record/1432200.

[4] ATLAS Collaboration, arXiv:1210.0435 [hep-ex].

[5] ATLAS Collaboration, ATLAS-CONF-2012-075, https://cdsweb.cern.ch/record/1460272.

[6] ATLAS Collaboration, arXiv:1207.6411 [hep-ex]. 